\title{
Locally Nilpotent Linear Groups
}

\author{
A. S. DETINKO AND D. L. FLANNERY
}

Dedicated to Martin L. Newell

\begin{abstract}
This article examines aspects of the theory of locally nilpotent linear groups. We also present a new classification result for locally nilpotent linear groups over an arbitrary field $\mathbb{F}$.
\end{abstract}

\section{Why Locally Nilpotent Linear Groups?}

Linear (matrix) groups are a commonly used concrete representation of groups. The first investigations of linear groups were undertaken in the second half of the 19th century, and currently linear group theory is a highly developed branch of group theory. In the past few decades interest in matrix groups has revived and increased, driven partly by the rapid development of computational group theory.

Locally nilpotent groups are a generalization of nilpotent groups. Over the years, many structural and classification results for locally nilpotent linear groups have been obtained. Further progress in the study of these groups is possible using computational techniques.

Group theoretical algorithms take as input a finite generating set for a group. The celebrated 'Tits alternative' states that a finitely generated linear group $G$ either is solvable-by-finite (that is, $G$ contains a normal solvable subgroup of finite index), or $G$ contains a nonabelian free subgroup. For linear groups of the latter type, some basic computational problems, such as membership testing and the conjugacy problem, are undecidable in general. Nilpotent linear groups on the other hand are solvable-by-finite and so are more suitable for computation (note that the class of nilpotent-by-finite linear

2000 Mathematics Subject Classification. Primary 20F19, Secondary 20H20.

This publication has emanated from research conducted with the financial support of Science Foundation Ireland. 
groups - which includes all locally nilpotent linear groups - forms an important subclass of

This point is underlined by Gromov's result [5], which implies that a finitely generated group has polynomial growth if and only if it is nilpotent-by-finite: hence, as explained in [1], certain algorithmic efficiency problems can be successfully overcome for locally nilpotent linear groups.

Another motivation for further study of locally nilpotent linear groups lies in possible application to abstract group theory concerns. Here an example is recent work [11] on the Carter conjecture for finite groups. Another example is the almost crystallographic groups, which are nilpotent-by-finite and arise naturally as linear groups over $\mathbb{Q}$ (see $[4, \S 5.3]$ ). Note that a finitely generated nilpotent group is polycyclic and so isomorphic to a $\operatorname{subgroup}$ of $\operatorname{GL}(n, \mathbb{Z})$ for some $n$; hence algorithms for nilpotent subgroups of $\operatorname{GL}(n, \mathbb{Q})$ serve as a key step toward algorithms for abstract finitely generated nilpotent groups.

\section{Structure of Locally Nilpotent Linear Groups}

Research into locally nilpotent linear groups relies heavily on knowledge of the structure of such groups. Systematic study of the structure of locally nilpotent linear groups was carried out by D. A. Suprunenko, beginning in the late 1940s [14]. Among other things, Suprunenko classified the maximal locally nilpotent subgroups of $\operatorname{GL}(n, \mathbb{F})$ when $\mathbb{F}$ is algebraically closed. Various authors extended some of Suprunenko's results to other fields. In particular, criteria for finiteness of the number of conjugacy classes of maximal locally nilpotent subgroups of $\mathrm{GL}(n, \mathbb{F})$, as well as classification of such groups in some partial cases, have been obtained from a detailed description of the structure of locally nilpotent linear groups over an arbitrary field (see e.g. [8]).

In the rest of this section we outline some of the most important structural results for locally nilpotent linear groups. A natural point of focus is the maximals, because each locally nilpotent subgroup of $\mathrm{GL}(n, \mathbb{F})$ is contained in a maximal locally nilpotent subgroup (by way of contrast, note that a nilpotent subgroup of $\mathrm{GL}(n, \mathbb{F})$ may not be contained in a maximal nilpotent subgroup).

We proceed via a standard reduction scheme: reducible $\rightarrow$ completely reducible $\rightarrow$ irreducible $\rightarrow$ absolutely irreducible $\rightarrow$ primitive. 
2.1. Reducible Locally Nilpotent Linear Groups. We use standard terminology for linear groups, as in $[14,15]$. A reducible subgroup $G$ of $\mathrm{GL}(n, \mathbb{F})$ is conjugate to a group of block upper triangular matrices, where the diagonal blocks form the irreducible parts of $G$, which are irreducible representations of $G$ over $\mathbb{F}$ in smaller degree. If $G$ is indecomposable locally nilpotent then a stronger statement holds: the irreducible parts of $G$ are pairwise equivalent ([15, p.223, Theorem 2]), so that $G$ is conjugate to a group of block upper triangular matrices

$$
\left(\begin{array}{cccc}
a(g) & a_{12}(g) & \cdots & a_{1 k}(g) \\
0 & a(g) & \cdots & a_{2 k}(g) \\
\vdots & \vdots & \ddots & \vdots \\
0 & 0 & \cdots & a(g)
\end{array}\right), g \in G
$$

where $a(G)=\{a(g) \mid g \in G\} \leq \mathrm{GL}(m, \mathbb{F})$ is irreducible locally nilpotent for some $m$ dividing $n$, and $a_{i j}(g) \in \operatorname{Mat}(m, \mathbb{F})$. If $\mathbb{F}$ is a perfect field then $a_{i j}(g)=c_{i j}(g) a(g)$ where $c_{i j}(g) \in \mathrm{C}_{\mathrm{Mat}(m, \mathbb{F})}(a(G))$; that is, $G$ is contained in the direct product of a completely reducible group over $\mathbb{F}$ with equivalent irreducible parts, and a unitriangular group over a division algebra. This reduces study of locally nilpotent linear groups to the irreducible case.

Another way to obtain a reduction to the completely reducible case is to use the Jordan decomposition. For each $g \in \mathrm{GL}(n, \mathbb{F})$ there is a unique unipotent matrix $g_{u} \in \mathrm{GL}(n, \overline{\mathbb{F}})$ and a unique diagonalizable matrix $g_{d} \in \mathrm{GL}(n, \overline{\mathbb{F}})$ such that $g=g_{d} g_{u}=g_{u} g_{d}$ (here $\overline{\mathbb{F}}$ is the algebraic closure of $\mathbb{F}$ ); see $[16$, p. 91, 7.2]. If $\mathbb{F}$ is perfect then $g_{u}$ and $g_{d}$ are both in $\operatorname{GL}(n, \mathbb{F})$.

Theorem 2.1. ([16, p.97, 7.11] and [15, p.240, Theorem 6]) Let $G \leq$ $\mathrm{GL}(n, \mathbb{F})$ be locally nilpotent, and define $G_{u}=\{g \in G \mid g$ unipotent $\}$, $G_{d}=\{g \in G \mid g$ diagonalizable $\}$. Then $G_{u}$ and $G_{d}$ are normal subgroups of $G$, and $\left\langle G_{u}, G_{d}\right\rangle=G_{u} \times G_{d}$.

The theorem implies that if $G$ is a completely reducible locally nilpotent subgroup of $\operatorname{GL}(n, \mathbb{F})$ then every subgroup of $G$ is completely reducible, and in particular every element of $G$ is diagonalizable $([16$, p.98, 7.12] and $[15$, p. 239, Theorem 5]).

Corollary 2.2. ([16, p.98, 7.13]) If $G \leq \mathrm{GL}(n, \mathbb{F})$ is locally nilpotent, and if for each $g \in G$ we have $g_{u}, g_{d} \in G$, then $G=G_{u} \times G_{d}$. 
The hypothesis $g_{u}, g_{d} \in G$ for all $g \in G$ is satisfied if $\mathbb{F}$ is finite. This is a partial case of the following.

Theorem 2.3. ([12, p. 136, Proposition 3]) Let $\mathbb{F}$ be perfect and $G$ be nilpotent. Define $\hat{G}_{u}=\left\{g_{u} \mid g \in G\right\}$ and $\hat{G}_{d}=\left\{g_{d} \mid g \in G\right\}$. Then $\hat{G}_{u}, \hat{G}_{d}$ are subgroups of $\operatorname{GL}(n, \mathbb{F})$, and $G \leq \hat{G}_{u} \times \hat{G}_{d}$.

The group $\hat{G}_{d}$ defined in Theorem 2.3 is completely reducible over $\mathbb{F}$.

2.2. Irreducible locally nilpotent linear groups. Each irreducible maximal locally nilpotent subgroup of $\mathrm{GL}(n, \mathbb{F})$ can be thought of as an absolutely irreducible maximal locally nilpotent subgroup of $\mathrm{GL}(m, \mathbb{E})$ for some $m$ dividing $n$ and field $\mathbb{E} \supseteq \mathbb{F}$ (see $[15$, p. 217 , Theorem 4]). This affords a reduction to the absolutely irreducible case, particularly in the classification of irreducible maximal locally nilpotent linear groups. Further investigation is possible in two directions, which are not mutually exclusive: reduction to $p$-subgroups of $\operatorname{PGL}(n, \mathbb{F})$, and reduction to primitive groups. The former is based on the following theorem.

Theorem 2.4. ([15, pp.220-221, Theorems 8, 9])

(i) Let $n=p_{1}^{a_{1}} \cdots p_{k}^{a_{k}}$ be the prime factorisation of $n$, where the $p_{i}$ are pairwise distinct primes. If $G$ is a maximal absolutely irreducible locally nilpotent subgroup of $\mathrm{GL}(n, \mathbb{F})$ then $G=$ $G_{1} \otimes \cdots \otimes G_{k}$ where $G_{i} \leq \mathrm{GL}\left(p_{i}^{a_{i}}, \mathbb{F}\right)$ is maximal absolutely irreducible locally nilpotent, $1 \leq i \leq k$.

(ii) Let $p$ be a prime. An absolutely irreducible subgroup $G$ of $\mathrm{GL}\left(p^{a}, \mathbb{F}\right)$ containing $\mathbb{F}^{\times} 1_{p^{a}}$ is a maximal absolutely irreducible locally nilpotent subgroup of $\mathrm{GL}\left(p^{a}, \mathbb{F}\right)$ if and only if $G / \mathbb{F}^{\times} 1_{p^{a}}$ is a Sylow p-subgroup of $\operatorname{PGL}\left(p^{a}, \mathbb{F}\right)$.

Irreducible locally nilpotent linear groups are center-by-periodic; in fact, the central quotient of each is a direct product of $p$-groups (see [13, Corollary 3.2.4]).

Except when $\mathbb{F}$ is finite or algebraically closed, the description of Sylow $p$-subgroups of $\operatorname{PGL}(n, \mathbb{F})$ is quite different to the description of Sylow $p$-subgroups of $\mathrm{GL}(n, \mathbb{F})$.

Sylow $p$-subgroups of $\operatorname{PGL}(n, \mathbb{F})$ were considered in [6], mainly for $p>2$. Classifying the Sylow 2-subgroups of $\operatorname{PGL}(n, \mathbb{F})$ is difficult. 
In [6], $p$-subgroups of $\operatorname{PGL}(n, \mathbb{F})$ are handled using the same techniques as for locally nilpotent linear groups, including the reduction to primitives.

The reduction to primitives is not so straightforward for locally nilpotent linear groups as it is for some other classes of linear groups, such as solvable groups. To appreciate this disparity, note that an irreducible imprimitive solvable subgroup of $\operatorname{GL}(n, \mathbb{F})$ is conjugate to a subgroup of $G \imath T$ where $G$ is a primitive solvable linear group and $T$ is a transitive solvable permutation group ([15, p.129, Theorem 5]); however, the wreath product of a locally nilpotent linear group and a nilpotent permutation group need not even be (locally) nilpotent.

A discussion of techniques for studying nilpotent primitive subgroups $G$ of $\mathrm{GL}(n, \mathbb{F})$ can be found in [8, Section 2]. One technique is to use the series $G \geq H \geq K \geq 1$ where $K=[G, G]$ and $H=\mathrm{C}_{G}(K)$.

A basic result here is as follows.

Theorem 2.5. ([8, Theorem 2]) Let $G \leq \mathrm{GL}\left(p^{a}, \mathbb{F}\right)$ be primitive absolutely irreducible locally nilpotent, $p \neq \operatorname{char} \mathbb{F}$. Then $K$ is an abelian p-group, $\Sigma=\langle K\rangle_{\mathbb{F}}$ is a field, $G / H \cong \operatorname{Gal}(\Sigma / \mathbb{F}),[H, H] \leq$ $\mathbb{F}^{\times} 1_{p^{a}}$, and $H$ is an absolutely irreducible primitive class 2 nilpotent subgroup of $\mathrm{GL}(m, \Sigma), m=p^{a} /|\Sigma: \mathbb{F}|$.

The primitive nilpotent subgroups of $\operatorname{GL}(n, \mathbb{F})$ for finite fields $\mathbb{F}$ have been completely classified, in [3] .

The paper [8] also summarizes results and methods for classifying maximal locally nilpotent subgroups of $\mathrm{GL}(n, \mathbb{F})$ over an arbitrary field $\mathbb{F}$.

Considerable attention has been paid to the problem of determining when the number of GL $(n, \mathbb{F})$-conjugacy classes of maximal locally nilpotent subgroups of $\mathrm{GL}(n, \mathbb{F})$ is finite. Finiteness of that number depends on finiteness of the groups $\mathbb{F}^{\times} /\left(\mathbb{F}^{\times}\right)^{m}$, for $m$ dividing $n$.

Groups over an algebraically closed field have been the most intensively studied.

Theorem 2.6. ([14, Chapter III $]$ and $[15$, Chapter VII $]$ ) Let $\mathbb{F}$ be algebraically closed.

(i) Irreducible locally nilpotent subgroups of $\mathrm{GL}(n, \mathbb{F})$ exist if and only if char $\mathbb{F}=0$ or char $\mathbb{F}$ does not divide $n$, in which cases there exists an irreducible nilpotent subgroup of $\mathrm{GL}(n, \mathbb{F})$ of nilpotency class $l$, for each and every nilpotency class $l \geq 1$. 
(ii) Irreducible maximal locally nilpotent subgroups of $\mathrm{GL}(n, \mathbb{F})$ are monomial and pairwise conjugate.

The matrix form of the groups in (ii) is given in [14, Chapter III, §7]. Note that (ii) implies that a completely reducible locally nilpotent linear group over an algebraically closed field is monomial.

In summary, locally nilpotent linear groups constitute a wellstudied class of groups, for which a lot of structural information and efficient methods of investigation are known. However the theory still has significant gaps. Most results deal only with absolutely irreducible maximal locally nilpotent subgroups of $\mathrm{GL}(n, \mathbb{F})$. Those results do not readily yield analogous results for locally nilpotent linear groups that are not maximal or are not absolutely irreducible (cf. [3]). Complete classifications of locally nilpotent subgroups of $\mathrm{GL}(n, \mathbb{F})$ are feasible only by placing restrictions on the field $\mathbb{F}$ or the degree $n$. In the sequel we allow arbitrary fields but restrict the degree.

\section{Prime Degree Locally Nilpotent Linear Groups}

We now give an illustration of how techniques in the theory of locally nilpotent linear groups may be applied to obtain a full classification in that theory. Specifically, in this section we classify the irreducible maximal locally nilpotent subgroups of $\operatorname{GL}(q, \mathbb{F})$, where $q$ is prime and $\mathbb{F}$ is any field. This classification is in the form of a complete list of $\operatorname{GL}(q, \mathbb{F})$-conjugacy class representatives of the groups, with each listed group defined by a generating set of matrices. Also we provide criteria to decide conjugacy between listed groups. Restricting to prime degree has several advantages: an irreducible subgroup $G$ of $\operatorname{GL}(q, \mathbb{F})$ is either abelian or absolutely irreducible, and is either primitive or monomial. Additionally, if $G$ is absolutely irreducible locally nilpotent then lies in a Sylow $q$-subgroup of $\operatorname{PGL}(q, \mathbb{F})$, and Sylow $q$-subgroups of $\operatorname{PGL}(q, \mathbb{F})$ have a simpler description than do Sylow subgroups of $\operatorname{PGL}(n, \mathbb{F})$ for composite degree $n$.

Certainly, a partial classification of the irreducible maximal locally nilpotent subgroups of $\mathrm{GL}(q, \mathbb{F})$ can be derived from a description of the absolutely irreducible maximal locally nilpotent subgroups of $\operatorname{GL}\left(q^{a}, \mathbb{F}\right)$. However, here we propose other methods and give a complete, self-contained result, which can be extended to get a complete classification in prime power degrees $q^{a}$. In particular we give an exact description of the Sylow 2 -subgroups of $\operatorname{PGL}(2, \mathbb{F})$, 
omitted by other authors. This is of special importance because (for example) classification of the Sylow 2-subgroups of $\operatorname{PGL}(n, \mathbb{F})$ in arbitrary degree $n$ depends on classification in degree 2 (cf. the case of 2-subgroups of $\mathrm{GL}(n, \mathbb{F})$ in $[7,10])$.

The methods used in this section were originally developed to classify the maximal irreducible periodic subgroups of $\operatorname{PGL}(q, \mathbb{F})$, in [2]. We begin by considering absolutely irreducible groups; abelian groups will be treated at the end.

By [15, Theorem 6, p. 217], GL $(q, \mathbb{F})$ contains absolutely irreducible locally nilpotent subgroups if and only if $\mathbb{F}^{\times}$has an element $\xi$ of order $q$ (of course char $\mathbb{F} \neq q$ ). Let $D=\left\{\operatorname{diag}\left(\beta \beta_{1}, \ldots, \beta \beta_{q}\right) \mid\right.$ $\left.\beta_{i} \in \operatorname{Syl}_{q}\left(\mathbb{F}^{\times}\right), \beta \in \mathbb{F}^{\times}\right\}$. For $\alpha \in \mathbb{F}^{\times}$, define

$$
I_{\alpha}=\left(\begin{array}{cc}
0 & 1_{q-1} \\
\alpha & 0
\end{array}\right) \in \operatorname{GL}(q, \mathbb{F})
$$

and write $I$ in place of $I_{1}$. For $H \leq \mathrm{GL}(q, \mathbb{F})$ let $\operatorname{Det}(H)=\{\operatorname{det}(h) \mid$ $h \in H\}$.

Assuming $\xi \in \mathbb{F}^{\times}$we define $H_{\alpha}=\left\langle D, I_{\alpha}\right\rangle$. The subgroup $H_{\alpha}$ of $\mathrm{GL}(q, \mathbb{F})$ is monomial and absolutely irreducible. Since $H_{\alpha} / \mathbb{F}^{\times} 1_{q}$ is a $q$-subgroup of $\operatorname{PGL}(q, \mathbb{F}), H_{\alpha}$ is locally nilpotent. If $\operatorname{Syl}_{q}\left(\mathbb{F}^{\times}\right)$is finite then $H_{\alpha}$ is nilpotent with nilpotency class $1+(q-1) \log _{q}\left|\operatorname{Syl}_{q}\left(\mathbb{F}^{\times}\right)\right|$.

Denote by $\pi$ the natural homomorphism from the group of all monomial matrices in $\operatorname{GL}(q, \mathbb{F})$ onto the group $\operatorname{Sym}(q)$ of $q \times q$ permutation matrices. The kernel of $\pi$ is the group $\mathrm{D}(q, \mathbb{F})$ of all diagonal matrices in $\operatorname{GL}(q, \mathbb{F})$.

Lemma 3.1. (Cf. $[2$, Lemma 20]) Let $a, b \in \mathrm{D}(q, \mathbb{F})$. The following statements are equivalent.

(i) Ia, Ib are $\mathrm{GL}(q, \mathbb{F})$-conjugate.

(ii) Ia, Ib are $\mathrm{D}(q, \mathbb{F})$-conjugate.

(iii) $\operatorname{det}(a)=\operatorname{det}(b)$.

Lemma 3.2. Let $H$ be an irreducible monomial locally nilpotent subgroup of $\mathrm{GL}(q, \mathbb{F})$. Then $H$ is conjugate in $\mathrm{GL}(q, \mathbb{F})$ to a subgroup of $H_{\alpha}$ for some $\alpha \in \mathbb{F}^{\times}$.

Proof. If $H$ is abelian then $\pi(H) \leq \operatorname{Sym}(q)$ is a transitive abelian group i.e. a cycle of order $q$, and $H \cap \mathrm{D}(q, \mathbb{F}) \leq \mathbb{F}^{\times} 1_{q}$. On the other hand if $H$ is absolutely irreducible then $H \mathbb{F}^{\times} / \mathbb{F}^{\times} 1_{q}$ is a $q$-group, so that $H \cap \mathrm{D}(q, \mathbb{F}) \leq D$, and $\pi(H)$ is again a cycle of order $q$. Hence up to conjugacy $H \leq\langle D, I a\rangle$ for some $a \in \mathrm{D}(q, \mathbb{F})$. Then $H$ is conjugate to a subgroup of $H_{\operatorname{det}(a)}$ by Lemma 3.1. 
Denote the set $\operatorname{Det}(D)=\operatorname{Syl}_{q}\left(\mathbb{F}^{\times}\right)\left(\mathbb{F}^{\times}\right)^{q}$ by $S$.

Lemma 3.3. If $\alpha \in S$ then $H_{\alpha}$ is $\mathrm{D}(q, \mathbb{F})$-conjugate to $H_{1}$. If $\alpha_{1}, \alpha_{2} \notin S$ then $H_{\alpha_{1}}$ and $H_{\alpha_{2}}$ are $\operatorname{GL}(q, \mathbb{F})$-conjugate if and only if $\operatorname{Det}\left(H_{\alpha_{1}}\right)=\operatorname{Det}\left(H_{\alpha_{2}}\right)$ i.e. $\left\langle\alpha_{1} S\right\rangle$ and $\left\langle\alpha_{2} S\right\rangle$ are identical subgroups of $\mathbb{F}^{\times} / S$ of order $q$.

Proof. Suppose $\alpha=\beta_{1} \beta^{q}$ for some $\beta_{1} \in \operatorname{Syl}_{q}\left(\mathbb{F}^{\times}\right)$and $\beta \in \mathbb{F}^{\times}$. Then $\operatorname{det}\left(I_{\alpha}\right)=\operatorname{det}(I b)$ where $b=\operatorname{diag}\left(\beta_{1} \beta, \beta, \ldots, \beta\right) \in D$. Therefore $H_{\alpha}$ is $\mathrm{D}(q, \mathbb{F})$-conjugate to $\langle I b, D\rangle=H_{1}$ by Lemma 3.1.

Now suppose $\alpha_{1}, \alpha_{2} \notin S$ and $\alpha_{1} \in\left\langle\alpha_{2}, S\right\rangle$. Then $\operatorname{det}\left(I_{\alpha_{1}}\right)=$ $\operatorname{det}\left(I_{\alpha_{2}}^{r} c\right)$ for some $c \in D$ and $1 \leq r \leq q-1$. Also there exists $x \in \operatorname{Sym}(q)$ such that $x I_{\alpha_{2}}^{r} c x^{-1}=I b$ for some $b \in \mathrm{D}(q, \mathbb{F})$. Hence by Lemma 3.1 once more, $H_{\alpha_{1}}$ and $H_{\alpha_{2}}$ are conjugate (this time by a monomial matrix).

Corollary 3.4. Define $\mathcal{H}=\left\{H_{\alpha} \mid \alpha \in \mathbb{F}^{\times} \backslash S\right\}$. The $\operatorname{GL}(q, \mathbb{F})$ conjugacy classes of the groups in $\mathcal{H}$ are in one-to-one correspondence with the distinct subgroups of $\mathbb{F}^{\times} / S$ of order $q$. Consequently the number of such classes is finite if and only if $\mathbb{F}^{\times} / S$ is finite.

Remark 3.5. If $\mathbb{F}$ is algebraically closed or finite then $\mathcal{H}$ is empty: a maximal absolutely irreducible monomial locally nilpotent subgroup of $\operatorname{GL}(q, \mathbb{F})$ is conjugate to $H_{1}$.

We turn next to primitive groups.

Lemma 3.6. Let $H$ be a primitive locally nilpotent subgroup of $\mathrm{GL}(q, \mathbb{F})$. Then $H$ has an irreducible abelian normal subgroup.

Proof. First we show that $H$ has an abelian noncentral normal subgroup. As $H$ is locally nilpotent, it is solvable, and thus has an abelian normal subgroup $A$ of finite index (see e.g. [15, p.135, Theorem 6]). If $A \leq \mathrm{Z}(H)$ then $H / \mathrm{Z}(H)$ is finite and thus $H$ is nilpotent. But a nonabelian nilpotent group certainly contains an abelian noncentral normal subgroup.

Any abelian noncentral normal subgroup $A$ of $H$ must be irreducible. For if $A$ were reducible then it would be diagonalizable with inequivalent irreducible parts, which contradicts primitivity of $H$.

Lemma 3.6 implies that a primitive locally nilpotent subgroup of $\operatorname{GL}(q, \mathbb{F})$ is contained in the $\operatorname{GL}(q, \mathbb{F})$-normalizer of the multiplicative group of a field extension $\Delta$ of $\mathbb{F} 1_{q}$ of degree $q$. Since this 
degree is prime, $\Delta$ is a cyclic extension of $\mathbb{F}$, with Galois group of order $q$. As long as $\mathbb{F}^{\times}$has an element $\xi$ of order $q, \Delta=\langle h\rangle_{\mathbb{F}}$ for some $h \in \mathrm{GL}(q, \mathbb{F})$ such that $h^{q}=\beta 1_{q} \in \mathbb{F}^{\times} 1_{q}$ by $[9$, p. 289, Theorem 6.2]. As $\beta \in\left(\mathbb{F}^{\times}\right)^{q}$ implies that $h$ is scalar, we have $\beta=\alpha^{r} \gamma^{q}$ for some $\alpha, \gamma \in \mathbb{F}^{\times}$and $1 \leq r \leq q-1$. Then $\gamma^{-1} h$ and $I_{\alpha}^{r}$ have the same characteristic (minimal) polynomial $X^{q}-\alpha^{r} 1_{q}$, and because $X^{q}-\alpha^{r} 1_{q}$ is $\mathbb{F}$-irreducible, $\gamma^{-1} h$ and $I_{\alpha}^{r}$ are conjugate. Hence $\Delta$ is conjugate to $\Delta_{\alpha}:=\left\langle I_{\alpha}\right\rangle_{\mathbb{F}}, \alpha \notin\left(\mathbb{F}^{\times}\right)^{q}$, and $\mathrm{N}_{\mathrm{GL}(q, \mathbb{F})}\left(\Delta_{\alpha}^{\times}\right)=\left\langle\Delta_{\alpha}^{\times}, d\right\rangle$ where $d=\operatorname{diag}\left(1, \xi, \ldots, \xi^{q-1}\right)$. Denote by $G(\alpha, b)$ the subgroup $\left\langle A_{\alpha}, d b\right\rangle$ of $\left\langle\Delta_{\alpha}^{\times}, d\right\rangle$, where $A_{\alpha} \supseteq \mathbb{F}^{\times} 1_{q}, A_{\alpha} / \mathbb{F}^{\times} 1_{q}$ is the Sylow $q$-subgroup of $\Delta_{\alpha}^{\times} / \mathbb{F}^{\times} 1_{q}$, and $b \in \Delta_{\alpha}^{\times}$. Since $A_{\alpha}$ is a noncentral irreducible subgroup, $G(\alpha, b)$ is absolutely irreducible.

Lemma 3.7. An absolutely irreducible primitive locally nilpotent subgroup $H$ of $\mathrm{GL}(q, \mathbb{F})$ is conjugate to a subgroup of some $G(\alpha, b)$.

Proof. Up to conjugacy $H=\left\langle H \cap \Delta_{\alpha}^{\times}, d b\right\rangle$ for some $\alpha \in \mathbb{F}^{\times} \backslash\left(\mathbb{F}^{\times}\right)^{q}$ and $b \in \Delta_{\alpha}^{\times}$. Then $H \cap \Delta_{\alpha} \leq A_{\alpha}$ by Theorem 2.4.

We use the notation $\varepsilon_{k}$ to stand for an element of multiplicative order $2^{k}$ in the algebraic closure of $\mathbb{F}$. If $k=2$ then we drop the subscript; that is, $\varepsilon$ is a square root of -1 .

Lemma 3.8. Suppose $\mathbb{F}$ does not have characteristic 2 , and $\varepsilon \notin \mathbb{F}$. Let $\mathbb{E}=\mathbb{F}(\varepsilon)$, and let $\sigma$ be the $\mathbb{F}$-involution of $\mathbb{E}$. If $\operatorname{Syl}_{2}\left(\mathbb{E}^{\times}\right)=\left\langle\varepsilon_{m}\right\rangle$ is cyclic then $\operatorname{Syl}_{2}\left(\mathbb{E}^{\times} / \mathbb{F}^{\times}\right)$is cyclic. Explicitly, one of the following must be true:

(i) $\sigma\left(\varepsilon_{m}\right)=-\varepsilon_{m}^{-1}$, and $\operatorname{Syl}_{2}\left(\mathbb{E}^{\times} / \mathbb{F}^{\times}\right)=\left\langle\varepsilon_{m} \mathbb{F}^{\times}\right\rangle$of order $2^{m-1}$; or

(ii) $\sigma\left(\varepsilon_{m}\right)=\varepsilon_{m}^{-1}$, and $\operatorname{Syl}_{2}\left(\mathbb{E}^{\times} / \mathbb{F}^{\times}\right)=\left\langle\left(1+\varepsilon_{m}\right) \mathbb{F}^{\times}\right\rangle$of order $2^{m}$.

Proof. We make some preliminary observations. First, either $\sigma\left(\varepsilon_{m}\right)=$ $\varepsilon_{m}^{-1}$ or $\sigma\left(\varepsilon_{m}\right)=-\varepsilon_{m}^{-1}$. Suppose $\sigma\left(\varepsilon_{k}\right)=\varepsilon_{k}^{-1}$. Then

$\sigma\left(\left(1+\varepsilon_{k}\right)^{2^{k}}\right)=\sigma\left(1+\varepsilon_{k}\right)^{2^{k}}=\left(1+\varepsilon_{k}^{-1}\right)^{2^{k}}=\left(\frac{1+\varepsilon_{k}}{\varepsilon_{k}}\right)^{2^{k}}=\left(1+\varepsilon_{k}\right)^{2^{k}}$.

Thus $\left(1+\varepsilon_{k}\right)^{2^{k}} \in \mathbb{F}$ and so

$$
\left(1+\varepsilon_{k}\right) \mathbb{F}^{\times} \in \operatorname{Syl}_{2}\left(\mathbb{E}^{\times} / \mathbb{F}^{\times}\right)
$$

if $k \geq 2$. Also, if $k>2$ then $\varepsilon_{k}^{-1}\left(\varepsilon_{k-1}+1\right)=\varepsilon_{k}^{-1}\left(\varepsilon_{k}^{2}+1\right)=\varepsilon_{k}+\varepsilon_{k}^{-1} \in$ $\mathbb{F}^{\times}$and so

$$
1+\varepsilon_{k-1} \in\left\langle\varepsilon_{k}\right\rangle \mathbb{F}^{\times} \text {. }
$$


Now let $x \mathbb{F}^{\times}$be a nontrivial element of $\operatorname{Syl}_{2}\left(\mathbb{E}^{\times} / \mathbb{F}^{\times}\right)$of order $2^{l}$, meaning that $x^{2^{l}} \in \mathbb{F}^{\times} \backslash\left(\mathbb{F}^{\times}\right)^{2}$.

Suppose $l=1$. Write $x=a+\varepsilon b, a, b \in \mathbb{F}$. Then $2 a b \varepsilon \in \mathbb{F}$ implies $a=0$ i.e.

$$
x \in\left\langle\varepsilon \mathbb{F}^{\times}\right\rangle \leq\left\langle\varepsilon_{m} \mathbb{F}^{\times}\right\rangle .
$$

Suppose $l \geq 2$. We have $\sigma(x)=y x$ for some $y \in \operatorname{Syl}_{2}\left(\mathbb{E}^{\times}\right)$, $y^{2^{l}}=1$. Then $y \neq-1$, because $y=-1$ implies $\left|x \mathbb{F}^{\times}\right|=2<2^{l}$. Further, $x=\sigma^{2}(x)=\sigma(y x)=\sigma(y) y x$ and so

$$
\sigma(y)=y^{-1} \text {. }
$$

As $\operatorname{tr}(x)=(1+y) x \in \mathbb{F}$,

$$
x \in(1+y)^{-1} \mathbb{F}^{\times}=(1+\sigma(y)) \mathbb{F}^{\times}=\left(1+y^{-1}\right) \mathbb{F}^{\times} .
$$

At last we are ready to complete the proof that either (i) or (ii) must be true. Let $\sigma\left(\varepsilon_{m}\right)=-\varepsilon_{m}^{-1}$; then $m>2$. By (3), we may take $l \geq 2$, in which event $4<|y| \leq 2^{m-1}$ by (4). Then $\left(1+y^{-1}\right) \mathbb{F}^{\times} \subseteq$ $\left\langle\varepsilon_{m}\right\rangle \mathbb{F}^{\times}$by (2), and by (5), (i) is proved.

If $\sigma\left(\varepsilon_{m}\right)=\varepsilon_{m}^{-1}$ then by $(1),\left\langle\left(1+\varepsilon_{m}\right) \mathbb{F}^{\times}\right\rangle \subseteq \operatorname{Syl}_{2}\left(\mathbb{E}^{\times} / \mathbb{F}^{\times}\right)$. Also $\varepsilon_{m} \mathbb{F}^{\times} \in\left\langle\left(1+\varepsilon_{m}\right) \mathbb{F}^{\times}\right\rangle^{2}$, since $\sigma$ fixes $\varepsilon_{m}^{-1}\left(1+\varepsilon_{m}\right)^{2}$. Part (ii) now follows from (2), (3), and (5).

Corollary 3.9. If $\operatorname{Syl}_{2}\left(\mathbb{E}^{\times}\right)$is quasicyclic then $\operatorname{Syl}_{2}\left(\mathbb{E}^{\times} / \mathbb{F}^{\times}\right)$is also quasicyclic, and $\operatorname{Syl}_{2}\left(\mathbb{E}^{\times} / \mathbb{F}^{\times}\right)=\left\{\left\langle\varepsilon_{k} \mathbb{F}^{\times}\right\rangle \mid \varepsilon_{k} \in \operatorname{Syl}_{2}\left(\mathbb{E}^{\times}\right)\right\}$.

Proof. For each $\varepsilon_{k} \in \operatorname{Syl}_{2}\left(\mathbb{E}^{\times}\right), \sigma\left(\varepsilon_{k}\right)=\varepsilon_{k}^{-1}$ i.e. $\sigma\left(\varepsilon_{k}\right)=-\varepsilon_{k}^{-1}$ is impossible. The corollary is then a consequence of Lemma 3.8 (ii) and (2) in the proof of the lemma.

Lemma 3.10. Let $|\mathbb{E}: \mathbb{F}|=q$ and $\mathbb{E}=\mathbb{F}(a)$, where $a^{q^{l}} \in \mathbb{F}$. Suppose $\xi \in \mathbb{F}^{\times}$, and $\mathbb{E} \neq \mathbb{F}(\varepsilon)$ if $q=2$. Then $\operatorname{Syl}_{q}\left(\mathbb{E}^{\times} / \mathbb{F}^{\times}\right)=\left\langle a \mathbb{F}^{\times}\right\rangle$.

Proof. If $q>2$, or $q=2$ and $\varepsilon \in \mathbb{F}$, then the lemma follows from [8, Lemma 2].

Let $q=2$ and $x \mathbb{F}^{\times} \in \mathbb{E}^{\times} / \mathbb{F}^{\times}$be of order $2^{m}, m \geq 1$, so that $x^{2^{m}}=\alpha$ for some $\alpha \in \mathbb{F}^{\times} \backslash\left(\mathbb{F}^{\times}\right)^{2}$. If $\alpha=-4 \gamma^{4}, \gamma \in \mathbb{F}^{\times}$, then $x^{2^{m-1}} / 2 \gamma^{2}$ is a square root of -1 , contradicting $\mathbb{E} \neq \mathbb{F}(\varepsilon)$.

Suppose $\alpha \notin-4\left(\mathbb{F}^{\times}\right)^{4}$. The polynomial $X^{4}-\alpha$ is $\mathbb{F}$-irreducible, so that if $m \geq 2$ then $|\mathbb{E}: \mathbb{F}| \geq 4$. Therefore $m=1, a=\sqrt{\beta}$ for some $\beta \in \mathbb{F}^{\times}$and $x=\sqrt{\alpha}$. For some $x_{1}, x_{2} \in \mathbb{F}$ we have $\sqrt{\alpha}=x_{1}+x_{2} \sqrt{\beta}$. Then $\alpha=x_{1}^{2}+\beta x_{2}^{2}+2 x_{1} x_{2} \sqrt{\beta}$ implies $x_{1}=0$ or $x_{2}=0$; as the latter is impossible we get $x \in\left\langle a \mathbb{F}^{\times} 1_{2}\right\rangle$ as required. 
Lemma 3.11. If $q>2$ or $\alpha \notin-\left(\mathbb{F}^{\times}\right)^{2}$ then $A_{\alpha}$ is the monomial group $\left\langle I_{\alpha}, \mathbb{F}^{\times} 1_{q}\right\rangle$; otherwise, $A_{\alpha}$ is primitive.

Proof. In Lemma 3.10 put $\mathbb{E}=\Delta_{\alpha}$ and $a=I_{\alpha}$. Then $A_{\alpha}=$ $\left\langle I_{\alpha}, \mathbb{F}^{\times} 1_{q}\right\rangle$ unless $q=2$ and $\Delta_{\alpha} \cong \mathbb{F}(\varepsilon)$ i.e. $\alpha \in-\left(\mathbb{F}^{\times}\right)^{2}$. If $A_{-\gamma^{2}}$ were monomial then $A_{-\gamma^{2}}^{2}$ would be in $\mathbb{F}^{\times} 1_{q}$; however $A_{-\gamma^{2}} / \mathbb{F}^{\times} 1_{q}$ contains the element $\left(1_{2}+\gamma^{-1} I_{-\gamma^{2}}\right) \mathbb{F}^{\times} 1_{q}$ of order 4 .

We refer to the set of hypotheses $q=2$ and $\alpha \in-\left(\mathbb{F}^{\times}\right)^{2}$ as case (*). Lemma 3.8 and Corollary 3.9 give an explicit description of the $A_{\alpha}$ in case $(*)$. Actually, a group $G\left(-\gamma^{2}, b\right)$ in this case is conjugate to some $G\left(-1, b^{\prime}\right)$, since $I_{-\gamma^{2}}$ is $\mathrm{D}(q, \mathbb{F})$-conjugate to $\gamma I_{-1}$ by Lemma 3.1. In all but case $(*),\left|G(\alpha, b) / \mathbb{F}^{\times} 1_{q}\right|=q^{2}$ (because $(d b)^{q}=\operatorname{det}(b) 1_{q}$ and $\left[I_{\alpha}, d\right]$ is scalar) and so $G(\alpha, b)$ is class 2 nilpotent. The group $G(-1, b)$ is locally nilpotent, and it is nilpotent only if $\operatorname{Syl}_{2}\left(\Delta_{-1}^{\times}\right)$is cyclic; then $G(-1, b) / \mathbb{F}^{\times} 1_{q}$ is a dihedral 2-group, and $G(-1, b)$ has nilpotency class $\log _{2}\left|\operatorname{Syl}_{2}\left(\Delta_{-1}^{\times}\right) / \mathbb{F}^{\times} 1_{2}\right|$.

Lemma 3.12. In case $(*), G(\alpha, b)$ is primitive. In all other cases, $G(\alpha, b)$ is primitive if and only if $\operatorname{det}(b) \notin\left\langle(-1)^{q-1} \alpha,\left(\mathbb{F}^{\times}\right)^{q}\right\rangle=$ $\operatorname{Det}\left(A_{\alpha}\right)$.

Proof. By Lemma 3.11, assume we are in a case other than $(*)$. Then [8, Lemma 1] yields that $G(\alpha, b)$ is primitive if and only if all elements of $G(\alpha, b)$ of order $q$ are scalar. Suppose $\operatorname{det}(b) \notin \operatorname{Det}\left(A_{\alpha}\right)$ and let $h \in G(\alpha, b),|h|=q$. If $h \notin A_{\alpha}$ i.e. $h=d b b_{1}, b_{1} \in A_{\alpha}$, then $h^{q}=\operatorname{det}\left(b b_{1}\right) 1_{q}$ implies that $\operatorname{det}(b) \in \operatorname{Det}\left(A_{\alpha}\right)$. Thus $h \in A_{\alpha}$, and $h$ is scalar by Lemma 3.11. Conversely, if $\operatorname{det}(b) \in \operatorname{Det}\left(A_{\alpha}\right)$ then for some $x \in A_{\alpha}, d b x$ is a nonscalar element of $G(\alpha, b)$ of order $q$.

Remark 3.13. Except in case $(*)$, if $\mathbb{F}$ is finite then $G(\alpha, b)$ is monomial.

Lemma 3.14. For $i=1,2$, let $g_{i}=d b_{i}$ where $b_{i} \in \Delta_{\alpha}$. The following statements are equivalent.

(i) $g_{1}$ and $g_{2}$ are $\mathrm{GL}(q, \mathbb{F})$-conjugate.

(ii) $g_{1}$ and $g_{2}$ are $\Delta_{\alpha}$-conjugate.

(iii) $\operatorname{det}\left(b_{1}\right)=\operatorname{det}\left(b_{2}\right)$.

Proof. See [2, Lemma 21].

Corollary 3.15. Apart from case $(*)$, primitive groups $G\left(\alpha, b_{1}\right)$, $G\left(\alpha, b_{2}\right)$ are conjugate if and only if $\operatorname{Det}\left(G\left(\alpha, b_{1}\right)\right)=\operatorname{Det}\left(G\left(\alpha, b_{2}\right)\right)$ and $\operatorname{det}\left(b_{1}\right)=\operatorname{det}\left(b_{2} c\right)$ for some $c \in A_{\alpha}$. 
Proof. Suppose $t G\left(\alpha, b_{1}\right) t^{-1}=G\left(\alpha, b_{2}\right)$. Since $t$ normalizes $A_{\alpha}$ as a consequence of Lemma 3.12, so $t \in\left\langle d, \Delta_{\alpha}^{\times}\right\rangle$. Then it can be checked that $t d b_{1} t^{-1} \in d b_{2} A_{\alpha}$. The other direction is clear by Lemma 3.14 .

Denote by $\mathcal{G}$ the set of all groups $G(\alpha, b)$ that are primitive, subject to the proviso that the only groups in case $(*)$ included in $\mathcal{G}$ are the $G(-1, b)$.

Remark 3.16. Note that $\mathcal{G}$ is empty if $\mathbb{F}$ is algebraically closed, for then $G(\alpha, b)$ is not defined. When $\mathbb{F}$ is finite, $\mathcal{G}$ is nonempty if and only if $q=2$ and $|\mathbb{F}| \equiv 3 \bmod 4$.

Lemma 3.17. If nonempty, the subset $\widetilde{\mathcal{G}}$ of $\mathcal{G}$ consisting of the groups not in case $(*)$ splits up into finitely many $\mathrm{GL}(q, \mathbb{F})$-conjugacy classes if and only if $\mathbb{F}^{\times} /\left(\mathbb{F}^{\times}\right)^{q}$ is finite.

Proof. If $\widetilde{\mathcal{G}}$ has only finitely many non-conjugate elements then $\mathbb{F}^{\times} /\left(\mathbb{F}^{\times}\right)^{q}$ is finitely generated and so finite. Conversely, if $\mathbb{F}^{\times} /\left(\mathbb{F}^{\times}\right)^{q}$ is finite then there are only finitely many subsets of $\mathbb{F}^{\times}$that are possibilities for $\operatorname{Det}(G(\alpha, b))$; hence the number of $\operatorname{GL}(q, \mathbb{F})$-conjugacy classes in $\widetilde{\mathcal{G}}$ is finite by Corollary 3.15 .

The next theorem is our main classification result.

Theorem 3.18. Suppose $\xi \in \mathbb{F}^{\times}$. A subgroup $G$ of $\mathrm{GL}(q, \mathbb{F})$ is an absolutely irreducible maximal locally nilpotent subgroup of $\mathrm{GL}(q, \mathbb{F})$ if and only if $G$ is conjugate to a group in $\mathcal{N}=\left\{H_{1}\right\} \cup \mathcal{H} \cup \mathcal{G}$, with the following exceptions when $q=2$ and $\varepsilon \notin \mathbb{F}$ :

(i) $H_{1}$ is a proper subgroup of $G(-1,1) \in \mathcal{G}$;

(ii) if $\alpha \notin-\left(\mathbb{F}^{\times}\right)^{2}$ and either $\operatorname{det}(b) \in-\left(\mathbb{F}^{\times}\right)^{2}$ or $\operatorname{det}(b) \in$ $\alpha\left(\mathbb{F}^{\times}\right)^{2}$, then $G(\alpha, b)$ is conjugate to a proper subgroup of $G(-1, c)$ where $\operatorname{det}(c)=\alpha$.

Proof. We have observed previously that all of the groups in $\mathcal{N}$ are absolutely irreducible locally nilpotent. By Lemmas 3.2, 3.3, and 3.7, and remarks after Lemma 3.11, an absolutely irreducible locally nilpotent subgroup of $\mathrm{GL}(q, \mathbb{F})$ is conjugate to a subgroup of a group in $\mathcal{N}$. Therefore it remains to show that the $H_{\alpha}$ and $G(\alpha, b) \in \mathcal{G}$ are really maximal locally nilpotent, apart from the stated exceptions.

Let $G$ be a maximal locally nilpotent subgroup of $\mathrm{GL}(q, \mathbb{F})$ containing $H_{\alpha}$. If $G$ is monomial then $t G t^{-1}=H_{\beta}$ for some $H_{\beta}$ and $t \in \mathrm{GL}(q, \mathbb{F})$. If $t D t^{-1} \neq D$ then $t D t^{-1} \cap D$ is scalar of index $q$ in $D$, 
so $\left|H_{\beta} / \mathbb{F}^{\times} 1_{q}\right|=q^{2}$; but $H_{\beta} / \mathbb{F}^{\times} 1_{q}$ has cardinality at least $q^{q+1}$. Thus $t D t^{-1}=D$, and then $q=\left|H_{\beta}: D\right| \geq\left|t H_{\alpha} t^{-1}: D\right|=\left|H_{\alpha}: D\right|=q$. Therefore $t H_{\alpha} t^{-1}=H_{\beta}$ i.e. $H_{\alpha}=G$. Now suppose $G$ is primitive, hence conjugate to some $G\left(\alpha_{1}, b\right)$. In a case other than (*) we have $\left|G / \mathbb{F}^{\times} 1_{q}\right|=q^{2}$, which is less than the cardinality of $H_{\alpha} / \mathbb{F}^{\times} 1_{q}$. Hence $q=2, \varepsilon \notin \mathbb{F}^{\times}, G$ is conjugate to $G(-1, b)$, and $H_{\alpha}=\left\langle d, I_{\alpha}, \mathbb{F}^{\times} 1_{2}\right\rangle$. Either $I_{\alpha}$ or $I_{-\alpha}=d I_{\alpha}$ is conjugate to nonscalar $h \in A_{-1}$ such that $h^{2} \in \mathbb{F}^{\times} 1_{2}$. Now $h$ has the form $\gamma I_{-1}, \gamma \in \mathbb{F}^{\times}$, and by comparing determinants we get $\alpha= \pm \gamma^{2}$. Thus if $H_{\alpha} \in \mathcal{H}$ then $H_{\alpha}$ is maximal. However $H_{1}=\left\langle d, I_{-1}, \mathbb{F}^{\times} 1_{2}\right\rangle$ is a proper subgroup of $G(-1,1)$.

Let $G$ be a maximal locally nilpotent subgroup of $\operatorname{GL}(q, \mathbb{F})$ containing $G(\alpha, b) \in \mathcal{G}$. For some $t \in \operatorname{GL}(q, \mathbb{F})$,

$$
t G t^{-1}=G\left(\alpha_{1}, b_{1}\right) \in \mathcal{G} .
$$

Apart from when $q=2, \varepsilon \notin \mathbb{F}^{\times}$, and $\alpha_{1}=-1$, we have

$$
\left|G(\alpha, b) / \mathbb{F}^{\times} 1_{q}\right|=\left|G\left(\alpha_{1}, b_{1}\right) / \mathbb{F}^{\times} 1_{q}\right|=\left|G / \mathbb{F}^{\times} 1_{q}\right|=q^{2},
$$

and thus $G(\alpha, b)=G$. Suppose now that $q=2, \varepsilon \notin \mathbb{F}$, and $\alpha_{1}=-1$. If $\alpha \in-\left(\mathbb{F}^{\times}\right)^{2}$ then $G(\alpha, b)$ is conjugate to some $G\left(-1, b_{2}\right)$, so that $G(\alpha, b)=G$. Therefore if $G(\alpha, b)$ is not maximal then $\alpha \notin-\left(\mathbb{F}^{\times}\right)^{2}$. For the rest of the proof $\alpha \notin-\left(\mathbb{F}^{\times}\right)^{2}$, which means that $G(\alpha, b)=$ $\left\langle I_{\alpha}, d b, \mathbb{F}^{\times} 1_{2}\right\rangle$. One of the following must occur: $t I_{\alpha} t^{-1} \notin A_{-1}$ and $t d b t^{-1} \in A_{-1}$, or $t I_{\alpha} t^{-1} \notin A_{-1}$ and $t d b t^{-1} \notin A_{-1}$.

In the first case, $\operatorname{det}(b) \in-\left(\mathbb{F}^{\times}\right)^{2}$. In the second case, the condition that $\left[I_{\alpha}, d b\right]$ is scalar forces $t d b t^{-1} \in I_{-1} t I_{\alpha} t^{-1} \mathbb{F}^{\times}$, so $\operatorname{det}(b) \in$ $\alpha\left(\mathbb{F}^{\times}\right)^{2}$. Suppose $\operatorname{det}(b) \in-\left(\mathbb{F}^{\times}\right)^{2}$. Then $\operatorname{det}(d b)=\operatorname{det}\left(\gamma I_{-1}\right)$ for some $\gamma \in \mathbb{F}^{\times}$. Since also $\operatorname{tr}(d b)=0=\operatorname{tr}\left(\gamma I_{-1}\right)$, there exists $s \in \mathrm{GL}(2, \mathbb{F})$ such that $s d b s^{-1}=\gamma I_{-1}$.

Since $I_{\alpha} d b I_{\alpha}^{-1}=-d b$, we have that $s I_{\alpha} s^{-1}$ normalizes $\Delta_{-1}^{\times}$. Hence $s I_{\alpha} s^{-1}=d c$, where $c \in \Delta_{-1}^{\times}$and $\operatorname{det}(c)=\alpha$. It follows that $G(\alpha, b)$ is conjugate to a proper subgroup of $G(-1, c)$. The reasoning that leads to this same conclusion in the case $\operatorname{det}(b) \in \alpha\left(\mathbb{F}^{\times}\right)^{2}$ is entirely similar.

With the next lemma we complete our classification of the irreducible maximal locally nilpotent subgroups of $\operatorname{GL}(q, \mathbb{F})$.

Lemma 3.19. Suppose $\operatorname{GL}(q, \mathbb{F})$ has irreducible abelian subgroups, and let $H$ be an irreducible maximal abelian subgroup of $\mathrm{GL}(q, \mathbb{F})$. 
(i) If $\xi \notin \mathbb{F}^{\times}$then $H$ is a maximal locally nilpotent subgroup of $\mathrm{GL}(q, \mathbb{F})$, and moreover any maximal locally nilpotent subgroup of $\mathrm{GL}(q, \mathbb{F})$ is abelian.

(ii) Let $\xi \in \mathbb{F}^{\times}$. Then $H$ is a maximal locally nilpotent subgroup of $\mathrm{GL}(q, \mathbb{F})$ unless $q=2$ and $\varepsilon \notin \mathbb{F}$. If $q=2$ and $\varepsilon \notin \mathbb{F}$ then $H$ is a maximal locally nilpotent subgroup of $\operatorname{GL}(q, \mathbb{F})$ if and only if $H / \mathbb{F}^{\times} 1_{2}$ is not a 2 -group.

Proof. so that if $H$ is not maximal locally nilpotent then $q=2$, $\varepsilon \notin \mathbb{F}$, and $H=A_{\alpha} \leq G(\alpha, \beta) \in \mathcal{G}$, by Lemma 3.11.

One situation in which all irreducible maximal locally nilpotent subgroups of $\operatorname{GL}(q, \mathbb{F})$ are nonabelian is when $q=2$ and $|\mathbb{F}|$ is a Mersenne prime.

Acknowledgment. This article is part of research carried out in the Department of Mathematics at NUI, Galway over a number of years. We are grateful to Professor Martin Newell for his ongoing generous support and encouragement of our work during this time.

\section{REFERENCES}

[1] R. Beals, Algorithms for matrix groups and the Tits alternative, J. Comput. System Sci. 58 (1999), 260-279.

[2] A. S. Detinko and D. L. Flannery, Periodic subgroups of projective linear groups in positive characteristic (submitted).

[3] A. S. Detinko and D. L. Flannery, Classification of nilpotent primitive linear groups over finite fields, Glasgow Math. J. 46 (2004), 585-594.

[4] B. Eick, Computational group theory, Jahresbericht der DMV 107, Heft 3 (2005), 155-170.

[5] M. L. Gromov, Groups of polynomial growth and expanding maps, Inst. Hautes Études Sci. Publ. Math. 53 (1981), 53-73.

[6] V. S. Konyukh, Sylow p-subgroups of a projective linear group, Vestsī Akad. Navuk BSSR Ser. Fìz.-Mat. Navuk (1985), no. 6, 23-29, 124-125.

[7] V. S. Konyukh, On linear p-groups, Vestsī Akad. Navuk BSSR Ser. Fīz.Mat. Navuk (1987), no. 1, 3-8, 124.

[8] V. S. Konyukh, Irreducible locally nilpotent linear groups, Fundam. Prikl. Mat. 4 (1998), 1345-1364.

[9] Serge Lang, Algebra, Addison-Wesley Publishing Co., Inc., Reading, Mass., 1993.

[10] C. R. Leedham-Green and W. Plesken, Some remarks on Sylow subgroups of general linear groups, Math. Z. 191 (1986), 529-535.

[11] A. Previtali, M. C. Tamburini, and E. P. Vdovin, The Carter subgroups of some classical groups, Bull. London Math. Soc. 36 (2004), 145-155.

[12] D. Segal, Polycyclic groups, Cambridge University Press, Cambridge, 1983. 
[13] M. Shirvani and B. A. F. Wehrfritz, Skew linear groups, London Mathematical Society Lecture Note Series, vol. 118, Cambridge University Press, Cambridge, 1986.

[14] D. A. Suprunenko, Soluble and nilpotent linear groups, Transl. Math. Monogr., vol. 9, American Mathematical Society, Providence, RI, 1963.

[15] D. A. Suprunenko, Matrix groups, Transl. Math. Monogr., vol. 45, American Mathematical Society, Providence, RI, 1976.

[16] B. A. F. Wehrfritz, Infinite linear groups, Springer-Verlag, Berlin, Heidelberg, New York, 1973.

A. S. Detinko and D. L. Flannery,

Department of Mathematics,

National University of Ireland,

Galway

alla.detinko@nuigalway.ie

dane.flannery@nuigalway.ie 